\title{
Designing Singlet Fission Candidates from Donor-Acceptor Copolymers
}

\author{
J. Terence Blaskovits ${ }^{\dagger}$, Maria Fumanal ${ }^{\dagger}$, Sergi Vela ${ }^{\dagger}$, and Clémence Corminboeuf $f^{\dagger, *}$ \\ 'Institute of Chemical Sciences and Engineering, École Polytechnique Fédérale de Lausanne (EPFL), Laboratory \\ for Computational Molecular Design, CH-1015 Lausanne, Switzerland. E-mail: clemence.corminboeuf@epfl.ch
}

\begin{abstract}
Singlet Fission (SF) has demonstrated significant promise for boosting the power conversion efficiency (PCE) of solar cells. Traditionally, SF is targeted as an intermolecular process, however its dependence on crystal packing makes molecular design difficult. In contrast, intramolecular SF (iSF) enables the exploration of tunable bi-chromophoric systems following well-defined structure-property relationships. In this work, we propose a set of parameters to screen conjugated donor-acceptor copolymer candidates with potential iSF behaviour. We focus our analysis on the $\mathrm{E}\left(\mathrm{S}_{1}\right)>2 \mathrm{E}\left(\mathrm{T}_{1}\right)$ thermodynamic condition and on the appropriate charge transfer (CT) character of $\mathrm{S}_{1}$. We map the $\mathrm{CT}$ character with respect to the frontier molecular orbital (FMO) energies of the constituent monomers, providing a cost-effective protocol for an accelerated screening of promising iSF donor-acceptor pairs, while minimizing the number of computations. These parameters are applied to a chemically diverse, curated library of 81 truncated dimers of synthetically feasible donor-acceptor copolymers. From our dataset, four candidates are flagged for iSF, two of which were previously experimentally reported. This protocol is envisioned to be scaled up for the high-throughput screening of large databases of donor-acceptor dimers for the design and identification of conjugated polymers capable of iSF.
\end{abstract}

\section{TOC GRAPHIC}

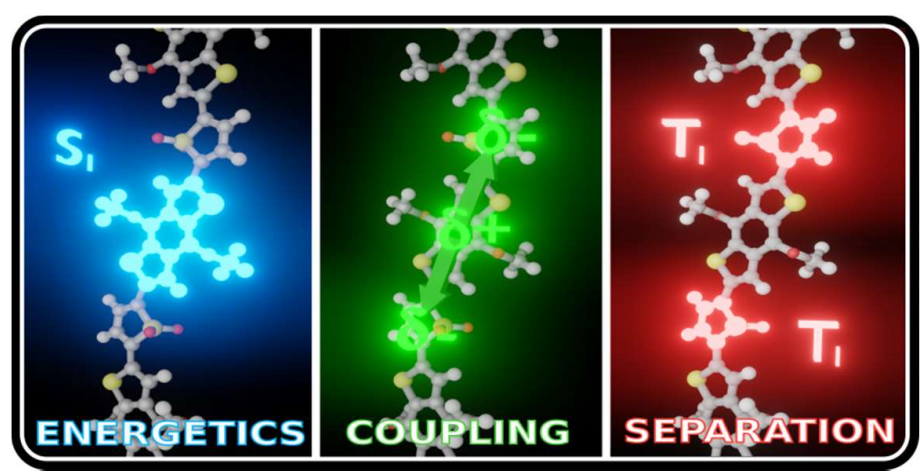




\section{INTRODUCTION}

First described in 1965, singlet fission (SF) is the spin-allowed conversion of a high-energy singlet to two lower-energy triplets. ${ }^{1}$ To be energetically possible, the excited singlet energy needs to be at least twice that of the triplet (i.e. $E\left(S_{1}\right) \geq 2 E\left(T_{1}\right)$ ). By definition, $S F$ is a multi-excitonic process: upon the absorption of light, the absorbing singlet splits into two independent triplets $\left(T_{1}\right)$ through a correlated triplet-triplet pair $\left({ }^{1} \mathrm{TT}\right)$ according to the following scheme: ${ }^{2}$

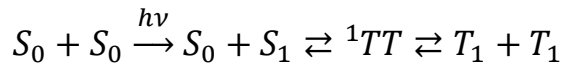

In organic photovoltaic devices, this theoretically leads to a doubled photocurrent if both excitons are separated at a donor-acceptor interface. In this way, materials exhibiting quantum efficiencies above $100 \%$ and power conversion efficiencies (PCE) beyond the thermodynamic (Shockley-Queisser) limit of 33\% become accessible. ${ }^{3}$

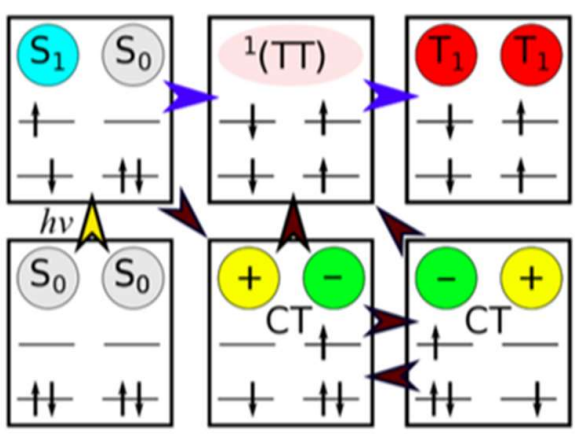

Figure 1. Mechanisms for SF after absorption: direct $S_{1}$ to ${ }^{1} \mathrm{TT}$ conversion following the blue arrows, or indirect conversion mediated by charge transfer (CT) states following the brown arrows.

SF involves two centers: following singlet excitation in one, there is energy transfer to the second, such that one triplet is formed at each center. ${ }^{2}$ Both direct $^{4}$ and charge-transfer ${ }^{5-8}$ mechanisms have been proposed for this (Figure 1). The formation of the triplet pair can proceed through either an intermolecular or an intramolecular process. In the former case, the centers are located on two separate molecules, while in the latter the two centers are covalently bound. Intermolecular SF has been extensively studied in molecular crystals. ${ }^{1,9-12}$ However, its success highly depends on the coupling between the separate units, ${ }^{13}$ which ultimately relies on the molecular packing, and as such can be difficult to predict and control. ${ }^{2,4}$ This limitation is circumvented in intramolecular SF (iSF).${ }^{14,15}$ Such is the case of covalently-linked dimers, in which synthetic modification of the linking units allows fine-tuning the spatial orientation between the sites. ${ }^{16-19}$ However, precisely due to the proximity of the two implicated fragments, the triplets in these systems recombine quickly and rarely become independent. Molecules with extended conjugation, such as polyenes and carotenoids, have also shown iSF, ${ }^{20}$ but their large structural flexibility makes nonradiative decay pathways readily available.

A few studies have demonstrated iSF in conjugated polymers, particularly in donor-acceptor copolymers, leading to some very promising candidates. ${ }^{21-25}$ On the one hand, Busby et al. designed a poly(benzodithiophene-alt-thiophene-1,1-dioxide) (BDT-TDO) copolymer with a triplet quantum yield of $170 \%$, which highlighted the importance of i) strong intramolecular donor-acceptor interactions, and ii) an acceptor core with a low triplet energy. On the other hand, Zhai et al. reported SF character in thin films of poly(phenylene-alt-vinylene) albeit not in solution, indicating that for certain polymers SF may involve inter-chain processes. ${ }^{23}$ Given the limited number of copolymer-based materials undergoing iSF reported so far, clear performance trends could not be established.

To date, research exploring the mechanisms of SF has been restricted to the small number of materials in which this process was experimentally observed. ${ }^{2,14,15,26}$ Computations of iSF in polymers have been done retroactively to rationalize SF reported in existing materials, ${ }^{21,27}$ but there has been a lack of effort to locate new iSF copolymer materials using computational tools. ${ }^{28}$ Only very recently a computational screening of intermolecular SF candidates, based on crystal structures, has been reported. ${ }^{26}$ Certainly, the discovery of novel iSF systems will largely benefit not only from large-scale screening but also from the development of new molecular design principles. In this work, we take advantage of the well-established modular chemistry of conjugated polymers, and their demonstrated 
potential for iSF, to explore their chemical space using computational screening techniques. Through systematic modulation of the donor and acceptor units in truncated dimers, we sought to establish design rules that link the monomer and dimer characteristics to the iSF potential of the resulting copolymer. In this way, we provide an accelerated computational screening framework that allows to explore a wide range of potential conjugated copolymers from in silico donor-acceptor combinations. From a curated database of 81 systems, we identify four promising iSF candidates; in two of these iSF has been previously reported. ${ }^{21,24}$ Altogether, we discuss both the rational and large-scale strategies of molecular design that will enable the discovery of new iSF materials.

\section{METHODOLOGY}

\subsection{Criteria to achieve iSF and Design Strategy}

The main conditions that SF candidates need to fulfill are the following: (1) the energy of $S_{1}$ is greater than or equal to twice the energy of $\mathrm{T}_{1}: E\left(S_{1}\right) \geq 2 E\left(T_{1}\right) ;{ }^{28}$

(2) the coupling between the two chromophores involved is strong, in order to promote $S_{1} \rightarrow{ }^{1} T T ;{ }^{14}$ and (3) the correlated triplet pair ( $\left.{ }^{1} \mathrm{TT}\right)$ must evolve into two independent triplets $\left(T_{1}\right)$ that can physically separate from one another and escape recombination. These criteria are referred to as the (1) energetic, (2) coupling and (3) separation criteria, respectively.

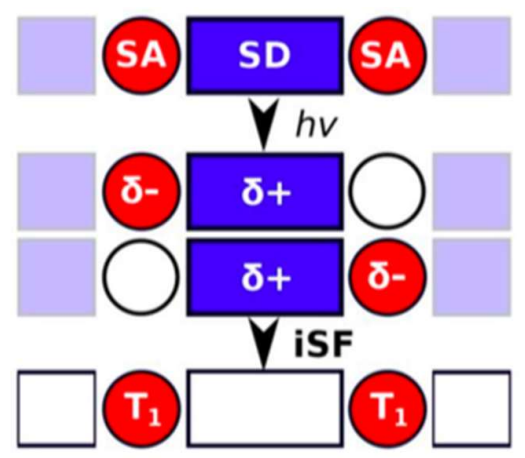

Figure 2. Fundamental design for strong donor-acceptortype iSF polymers, in which absorption leading to $S_{1}$ on the strong donor (SD, in blue) provides enough charge transfer character (denoted with $\delta^{+}$and $\delta$-) to efficiently generate local $\mathrm{T}_{1}$ on the adjacent strong acceptors (SA, in red). ${ }^{22}$
Within the framework of donor-acceptor copolymers, the design strategy consists in combining a donor core, which acts as the main photon absorption site and whose $S_{1}$ has a dominant $C T$ contribution to the acceptor, with an acceptor featuring a low lying triplet state (see Figure 2). ${ }^{24}$ In this way, the strong CT character of $\mathrm{S}_{1}$ is expected to promote an efficient splitting to ${ }^{1} \mathrm{TT}$ (coupling criterion), while the spatial separation between the two triplets on nearby acceptors, separated by the donor unit, is expected to diminish the possibility of triplet-triplet recombination (separation criterion).

\subsection{Database construction}

Our database includes nine donors and nine acceptors that are commonly found in the literature of conjugated polymers. ${ }^{29-31}$ These contain cyclic, fused and bridged derivatives of thiophene, benzene, pyrrole and other heterocycles (see Figure 3). Wellestablished chemical motifs were prioritized to ensure that potential SF candidates that emerge from this database are synthetically feasible, as well as units that are amenable to multiple polymerization techniques and that can be synthesized with high atom economy in few steps. ${ }^{32-34}$ Units reported in previous works to display iSF in conjugated polymers were included: thiophene-1,1-dixoide (TDO) ${ }^{24}$, benzodithiophene $(\mathrm{BDT})^{22}$, phenylene ${ }^{23}$, vinylene ${ }^{23}$ (in the form of (E)-2-(2-(thiophen-2-yl)vinyl)thiophene), TVT), cyclopentadithiophene $\quad(\text { CPDT })^{21}$, benzothiadiazole (BT) ${ }^{21}$ and isoindigo (iI). ${ }^{25}$

Each donor and acceptor core was encoded as a SMILES string. ${ }^{35}$ The dimer set was generated by linking the nine donors with the nine acceptors through a covalent carbon-carbon bond to form the 81 donor-acceptor pairs. The resulting SMILES strings of the dimer were then converted to cartesian coordinates using the gen3d operation in OpenBabel, ${ }^{36}$ which includes a conformational search and a geometry optimization at the force field level. Tighter convergence criteria were then applied by reoptimizing the geometries at the Density Functional Theory (DFT) level. The full method used for dataset construction is detailed in section $\mathrm{S} 1$, and all data are made available in the Materials Cloud repository. 


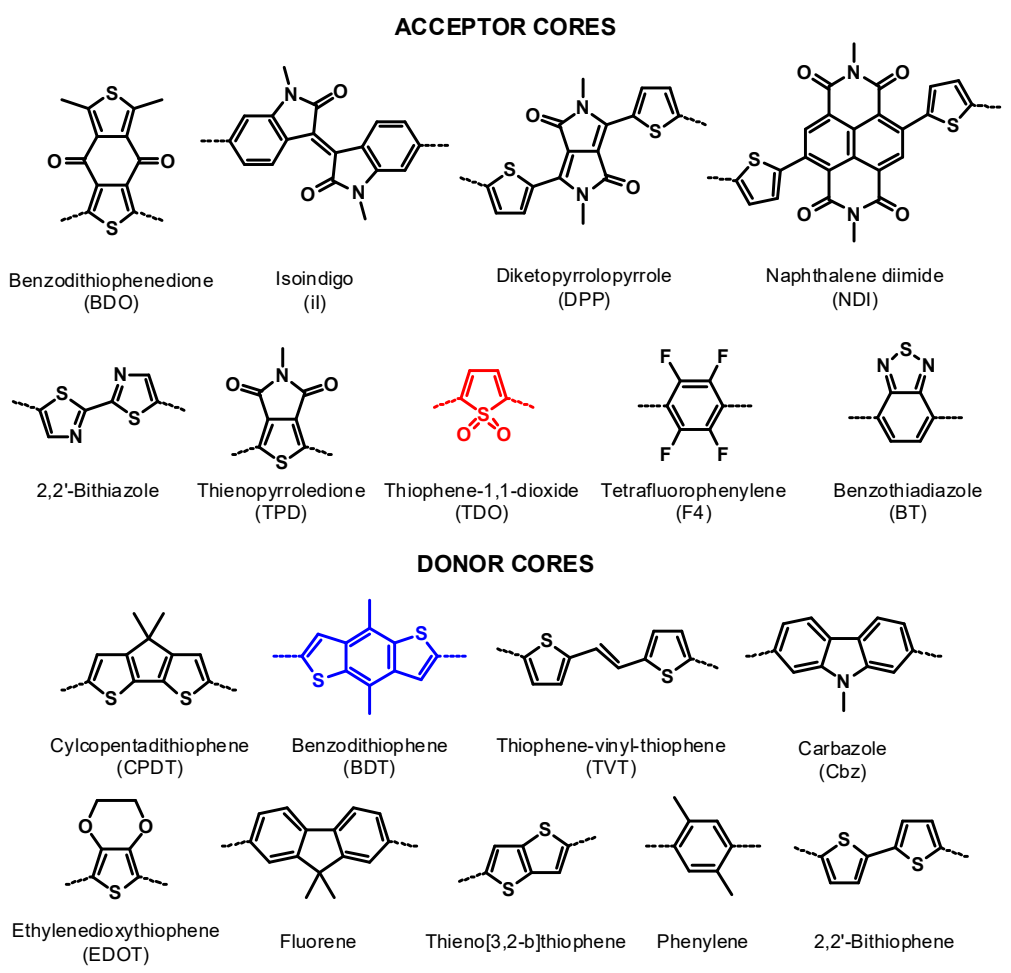

Figure 3: Library of acceptors and donors. Dotted lines denote the bonding positions for D-A polymer chains. Common abbreviations for the cores, when available, are given in brackets. Structures discussed in section 3.1 are highlighted in red and blue.

\subsection{Computational Details}

DFT computations were performed using the Gaussian16 package (Revision A.03) ${ }^{37}$ Ground state geometries were optimized at the $\omega \mathrm{B} 97 \mathrm{X}-\mathrm{D} / 6-31 \mathrm{G}^{*}$ level of theory. Normal mode analysis confirmed that the stationary points were minima with all real frequencies. To evaluate the energetic criterion, vertical excitations and excited state geometry optimizations were computed using time-dependent density functional theory (TD-DFT), within the Tamm-Dancoff approximation (TDA) to correct for the triplet instabilities reported in TD-DFT. ${ }^{38}$ The range-separated hybrid functional $\omega \mathrm{B} 97 \mathrm{X}-\mathrm{D}$ was used, given its accurate treatment of excited states, in particular with respect to its description of charge transfer character. ${ }^{39,40}$ Full details of functional and basis set benchmarking are given in section S2. For computations with solvent, the solvent cavity reaction field (SCRF) was used with a conductorlike polarizable continuum model (CPCM), which was found to give nearly equivalent results to the
SMD continuum model (see section S2.3 for comparison of solvent models).

Gaussian output files were parsed with cclib $^{41}$ and TheoDORE (version 1.7.2) ${ }^{42,43}$ to assess the coupling and separation criteria by means of the local and CT character of the electronic transitions. This is done through the $\Omega_{i j}$ values, which quantify the amount of hole $\left(\mathrm{h}^{+}\right)$and electron $\left(\mathrm{e}^{-}\right)$transition density located in the different molecular fragments $(i, j)$. In the present case, we considered two fragments: the donor (D) and the acceptor (A) cores of the donoracceptor dimer. Accordingly, the electronic transition is decomposed into a matrix containing four omega values, in which the diagonal elements $\left(\Omega_{\mathrm{D} \rightarrow \mathrm{D}} \quad\right.$ and $\left.\Omega_{\mathrm{A} \rightarrow \mathrm{A}}\right) \quad$ quantify intra-fragment contributions (i.e. the hole and electron are formed on the same fragment, $\mathrm{i}=\mathrm{j}$ ), while the off-diagonal elements are the charge-transfer components $(i \neq j)$, in which the electron density is transferred from the donor fragment to the acceptor fragment $\left(\Omega_{\mathrm{D} \rightarrow \mathrm{A}}\right)$ or vice versa $\left(\Omega_{\mathrm{A} \rightarrow \mathrm{D}}\right)$. For each transition, the sum of the four omega values is 1 . 


\section{RESULTS}

The Results are presented in four sections. In section 3.1. we analyse the excited state characteristics of the BDT-TDO copolymer, which previously exhibited iSF. In section 3.2 and 3.3 we establish specific numerical thresholds to efficiently screen the energetic, coupling and separation criteria (section 2.1) from a curated dataset of 81 donoracceptor dimers (section 2.2). Finally, in section 3.4 we map the CT character of $\mathrm{S}_{1}$ with respect to the frontier molecular orbital (FMO) energies of the constituent monomers, and test it on 25 substituted bithiophene-benzothiadiazole pairs.

\subsection{BDT-TDO copolymer}

Copolymers made of thiophene-1,1-dioxide (TDO, shown in red in Figure 3) acceptors, and benzodithiophene (BDT, shown in blue in Figure 3) donors have shown good SF quantum yields and triplet pair lifetimes. ${ }^{24}$ Being a prototypical SF copolymer with excellent properties, we selected it as a representative test-case to establish a costeffective computational strategy to evaluate iSF design criteria (section 2.1).

Vertical and adiabatic $S_{1}, T_{1}, T_{2}$ and $Q_{1}$ energies were computed at the $\omega \mathrm{B} 97 \mathrm{X}-\mathrm{D} / \mathrm{TZVP}$ level considering a D-A dimer and a D-A-D-A tetramer model of the BDT-TDO copolymer (see Table S3). While the energetic criterion (1) is not fulfilled for the dimer model at the Frank-Condon point $\left(\Delta E_{S T}^{v e r t}=E\left(S_{1}\right)-2 E\left(T_{1}\right)=-0.72 \mathrm{eV}\right)$, this value becomes much closer to zero in the adiabatic picture $\left(\Delta E_{S T}^{a d i a b}=-0.18 \mathrm{eV}\right)$, and even slightly positive for the extended tetramer model $\left(\Delta E_{S T}^{\text {adiab }}=\right.$ $0.04 \mathrm{eV})$. This highlights the impact of adiabaticity in predicting potential SF behaviour, while shows that the dimer-to-tetramer extension has a much less meaningful impact on the energetic criterion.

Fragment-based decomposition analysis of hole and electron density in the excited states revealed that charge transfer from the donor core (BDT) to the acceptor (TDO) at the Frank-Condon point is the primary contribution to the $S_{1}$ excitation $\left(\Omega_{\mathrm{D} \rightarrow \mathrm{A}}^{\mathrm{S} 1}=\right.$ 0.46 ), following the coupling criterion, whereas a local excitation within the acceptor dominates in $\mathrm{T}_{1}$ state $\left(\Omega_{\mathrm{A} \rightarrow \mathrm{A}}^{\mathrm{T} 1}=0.42\right)$, matching with the separation criterion (see section 2.1). Similar values were obtained for the adiabatic states and, in all cases, the three other possible contributions to excitation character are smaller (see Tables S4-S5).

In order to be efficient, SF needs to overcome triplet-triplet annihilation (TTA) paths, that is, recombination of the two $T_{1}$ states to higher excited states such as $\mathrm{T}_{2}$ or $\mathrm{Q}_{1}$. For the recombination paths to be energetically unfavourable, both $E\left(T_{2}\right)-$ $2 E\left(T_{1}\right)$ and $E\left(Q_{1}\right)-2 E\left(T_{1}\right)$ should be positive. ${ }^{14}$ While low-lying $T_{2}$ or $Q_{1}$ states do not necessarily prevent the singlet splitting, they may reduce the rate of SF. The computed adiabatic energy of $\mathrm{T}_{2}$ resulted in $0.4 \mathrm{eV}$ below $\mathrm{S}_{1}$ when evaluated in gas phase conditions. Remarkably, this difference is significantly reduced to $0.1 \mathrm{eV}$ when including polar solvent effects. This diminution originates in the strong CT character of $S_{1}$, which has negligible contributions to the mainly local $T_{1}$ or $T_{2}$ states. Finally, we found that $\mathrm{Q}_{1}$ is consistently above both $\mathrm{S}_{1}$ and $\mathrm{T}_{2}$ in all cases.

In summary, our computations correctly predict (1) thermodynamic adequacy, (2) D-to-A CT character as the largest contribution to $S_{1}$, and (3) $T_{1}$ being primarily localized on the acceptor in BDT-TDO copolymer. Adiabaticity plays an important role on $\Delta E_{S T}$ prediction and thus, empirical rules to correct cost-effective vertical energies of D-A dimers are required. In the next section, we exploit this approach using a curated database of D-A dimers.

\subsection{Excited States Energies}

Threshold for the energetic criterion. We sought to establish a computationally efficient method to evaluate $\Delta E_{S T}$ (i.e. the energetic criterion), which bypasses the structural optimization of $S_{1}$ and $T_{1}$. To do so, the $S_{1}$ and $T_{1}$ energies of 81 donor-acceptor dimers in our dataset (see section 2.2) were evaluated at the $\mathrm{S}_{0}$ geometry and at their excited state minima to establish an empirical trend. We found that the relationship between the vertical and adiabatic energies for both $S_{1}$ and $T_{1}$ is linear (see Figures S9 and S10), and thus the correlation between the vertical and adiabatic $\Delta E_{S T}$ is also linear (see Figures 4 and S11). The vertical $\mathrm{T}_{1}$ energies are 
found to be consistently higher than those obtained from adiabatic computations. As a result, all dimers with $\Delta E_{S T}>0 \mathrm{eV}$ in adiabatic computations are also above $-1 \mathrm{eV}$ when computed vertically (shown as dotted lines in Figure 4). It is therefore possible to approximate the energy conservation criterion computed adiabatically to:

$$
\Delta E_{S T}^{\text {adiab }} \geq 0 \mathrm{eV} \leftrightarrow \Delta E_{S T}^{\text {vert }} \geq-1 \mathrm{eV}
$$

This provides a simple and cheap method to estimate $\Delta E_{S T}$ from vertical computations by means of systematically shifting the threshold value corresponding to the energetic criterion. It is noted that the linear relationship between the vertical and adiabatic values fails when $\Delta E_{S T}^{\text {vert }}$ is below $-2 \mathrm{eV}$. However, this will not bias our identification of potential iSF candidates based on this criterion, as this loss of correlation occurs well below the established threshold of $-1 \mathrm{eV}$.

To assess if dimer models are representative of larger oligomeric (and polymeric) systems, we evaluated the vertical and adiabatic excited state energies of a subset of 21 tetramers. We considered systems that span the entire range of excitation energies and omega values, with each donor and acceptor moiety represented at least once. The correlation between the dimer and tetramer vertical excitation energies is linear for the entire range of excitation energies and the y-intercept is close to zero (see Figures S14-S15). Consequently, negligible deviations in $\Delta E_{S T}^{\text {vert }}$ and $\Delta E_{S T}^{\text {adiab }}$ are obtained (see Figures S16-S17).

Dihedral analysis of the conjugated dimers and tetramers. An important structural feature of conjugated copolymers is the degree of coplanarity and how it is affected in the excited state. To address this question we evaluated the variation in the dihedral angle $(\varphi)$ between the donor and acceptor units upon excited state geometry optimization. Comparison of the dihedral angles in the $S_{0}$ and $S_{1}$ minima reveal little changes upon adiabatic relaxation (see Figure S22), other than the dimers becoming slightly more coplanar $\left(\varphi \rightarrow 0^{\circ}\right.$ or $\left.180^{\circ}\right)$ at the $\mathrm{S}_{1}$ minimum in most cases. ${ }^{44}$ Consequently, the effective conjugation and character of the excited states remains very similar in the vertical and adiabatic pictures (see Figures S12-S13), indicating that the excited states character of the D-A dimers can be efficiently captured through vertical excitation computations, which are addressed in section 3.3.

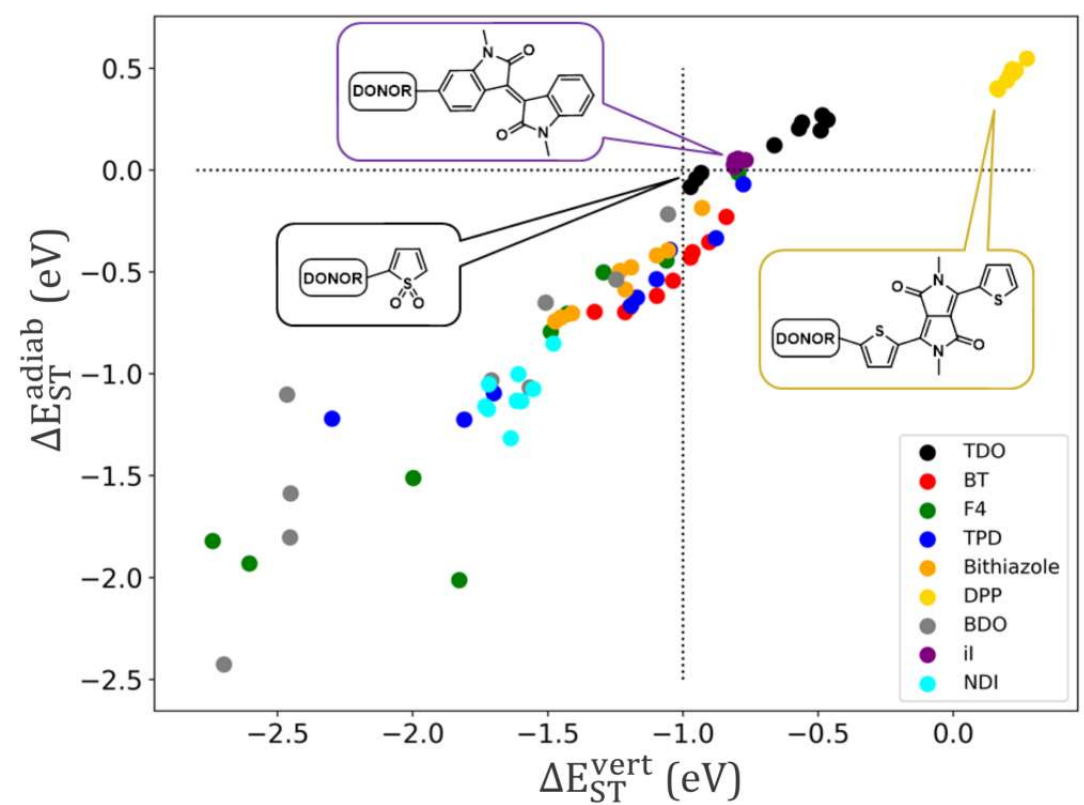

Figure 4: $\Delta E_{S T}^{\text {vert }}$ and $\Delta E_{S T}^{\text {adiab }}$ values associated with the 81 donor-acceptor dimers, coloured based on the acceptor. These are computed with TDDFT (TDA) at the $\omega \mathrm{B} 97 \mathrm{X}-\mathrm{D} / 6-31 \mathrm{G}^{*}$ level. The vertical and adiabatic cut-offs established as energy conservation criterion are shown as dotted lines at -1.0 and $0.0 \mathrm{eV}$, respectively. 
The dihedral angle between the central donor and acceptor units in the tetramers is virtually equivalent to those in the analogous dimers (see Figure S23). Interestingly, for most materials the change in this dihedral angle upon excited state optimization in $\mathrm{S}_{1}$ is smaller in the tetramer than in the dimer (see Figure S24). This observation suggests that the polymer chain extension restricts the dihedral angle change induced in the excited state. As a consequence, very similar excited state character is obtained in the adiabatic and vertical excitations (see Figures S18-S19), strengthening the assertion that vertical computations at the $\mathrm{S}_{0}$ geometry describe accurately the expected behaviour of larger adiabatic systems. Comparison between the excited state character of $S_{1}$ and $T_{1}$ in the dimer and tetramer shows only small deviations while following a nice linear trend (see Figures S20-S21). Considering the structural diversity of our library, these results demonstrate unequivocally that the dimer model, while representing a computational simplification, nonetheless offers an accurate picture of the excited state behaviour and iSF potential of larger donoracceptor polymeric systems.

Dependence of energy splitting on monomer structure. The computed $\Delta E_{S T}^{\text {vert }}$ and $\Delta E_{S T}^{\text {adiab }}$ values of the $81 \mathrm{D}$-A dimers are represented with respect to their acceptor unit in Figure 4. Considering the nine sets of same-acceptor pairs, the dimers can be classified into two categories: those in which the energetic criterion mainly depends on the acceptor unit (acceptor-dependent), and those which have a broad distribution of $\Delta E_{S T}$ depending on both the donor and acceptor constituents (donor-tuning). In the former category, all dimers containing the same acceptor (DPP, iI, TDO and NDI) have approximately the same energy splitting values regardless of the donor. In the latter category are the dimers containing BT, F4, TPD, bithiazole, and BDO acceptors, for which certain donors modulate the excited state energy levels towards favourable splitting. In particular, the donors TVT, CPDT, 2,2'bithiophene, thienothiophene and BDT, which all include thiophene moieties, shift $\Delta E_{S T}$ to more positive values, while the donors that do not have thiophene motifs (Cbz, fluorene and phenylene) are systematically detrimental to the energy conservation condition. Smaller values of $\Delta E_{S T}$ originate in non-planar dihedral angles between the donor and acceptor units in dimers linked via a benzene ring. This leads to a weaker effective conjugation and, generally, to higher $T_{1}$ excitation energies. A representative case is shown in Figure 5 for the TPD acceptor. The six donors that are coplanar with the acceptor $\left(\varphi \approx 0^{\circ}\right)$ can form intramolecular chalcogen bonds promoted by the thiophene unit, and the resulting dimer possess vertical energy splitting values between $-0.78 \mathrm{eV}$ and $-1.17 \mathrm{eV}$. In contrast, the D-A dimers that do not contain thiophene motifs in the donor exhibit higher dihedral angles and more negative $\Delta E_{S T}$ values.

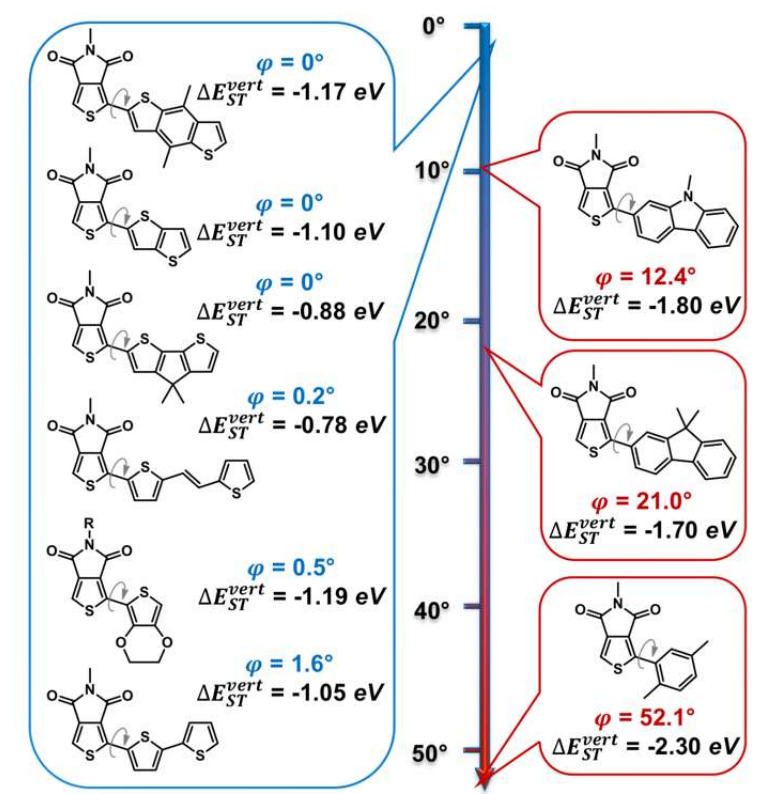

Figure 5: Influence of the dihedral angle $(\varphi)$ between the donor and acceptor cores on $\Delta E_{S T}^{v e r t}$ for the nine dimers containing the acceptor TPD at their respective $\mathrm{S}_{0}$ minima. The thiophene- and benzene-based donors are shown in blue and red, respectively.

\subsection{Excited State Character}

In order to identify how the excitation energies and thus, $\Delta E_{S T}$, are affected by the different state character of $S_{1}$ and $T_{1}$, we performed a fragmentbased analysis of the main local and CT contributions. We focused on the donor-to-acceptor CT component of $\mathrm{S}_{1}\left(\Omega_{\mathrm{D} \rightarrow \mathrm{A}}^{\mathrm{S1}}\right)$ and on the local acceptor contribution of $\mathrm{T}_{1} \quad\left(\Omega_{\mathrm{A} \rightarrow \mathrm{A}}^{\mathrm{T} 1}\right)$ as key 
requirements for efficient iSF that will potentially favour singlet splitting and prevent fast TTA, respectively (criteria 2 and 3 , section 2.1). These are represented for the 81 dimers in Figure 6. The dimers BDT-TDO (discussed in section 3.1) and CPDT-BT, for which iSF has been reported, ${ }^{21,24}$ fulfill both criteria, with the key contributions $\left(\Omega_{\mathrm{A} \rightarrow \mathrm{A}}^{\mathrm{T} 1}\right.$ and $\left.\Omega_{\mathrm{D} \rightarrow \mathrm{A}}^{\mathrm{S} 1}\right)$ both above 0.4 . For that reason, we tentatively select 0.4 as threshold for screening purposes. Remarkably, all other dimers found above this threshold have BT as acceptor, which systematically generates very promising candidates for iSF. In fact, the classification into acceptordependent and donor-tuning D-A dimers discussed for the energy splitting values remains valid for $\Omega_{\mathrm{A} \rightarrow \mathrm{A}}^{\mathrm{T} 1}$ and $\Omega_{\mathrm{D} \rightarrow \mathrm{A}}^{\mathrm{S} 1}$. In particular, DPP- and iI- dimers, which systematically show positive $\Delta E_{S T}$, lead to large $\Omega_{\mathrm{A} \rightarrow \mathrm{A}}^{\mathrm{T} 1}$ and small $\Omega_{\mathrm{D} \rightarrow \mathrm{A}}^{\mathrm{S} 1}$ in all cases (due to large $\Omega_{\mathrm{A} \rightarrow \mathrm{A}}^{\mathrm{S} 1}$ values). Therefore, the dimers involving these acceptors will not undergo iSF, but most likely inter-chain SF based on local acceptor states. Large singlet-triplet energy splitting has been previously associated with local excitations in organic systems. ${ }^{45}$ However, new design principles need to be considered when evaluating the iSF capabilities of D-A copolymers. From our results, it is possible to envision a 'modular' design strategy based on the frontier molecular orbital (FMO) energies of the donor and acceptor units to screen the CT or local character of $S_{1}$ in the dimer.

\subsection{Charge Transfer Prediction}

In this section, we correlate the CT character of $S_{1}$ in the 81 D-A dimers with the FMOs of the 18 constituent monomers (collected in Table S6). This is represented schematically in Figure 7, where it is shown how the $\mathrm{CT}$ excitation $(\mathrm{D} \rightarrow \mathrm{A})$ competes with local excitations in either the donor or the acceptor depending on the relative ordering of the FMOs. Within this approximation, the ratio between the local orbital gaps and the resulting CT energy difference defined as

$$
\frac{A_{L U M O}-A_{H O M O}}{A_{L U M O}-D_{H O M O}} \text { and } \frac{D_{L U M O}-D_{H O M O}}{A_{L U M O}-D_{H O M O}}
$$

will estimate favourable $(>1)$ or unfavourable $(<1)$ CT excitations with respect to local excitations. The approximation of considering orbital gaps as oneelectron transitions is possible because the relationship with the local excited energies is rather linear (see Figures S25 and S26). This shows that the exciton binding energy, defined as the difference between the orbital gap and the excitation energies, is relatively constant for all donors and acceptors considered.

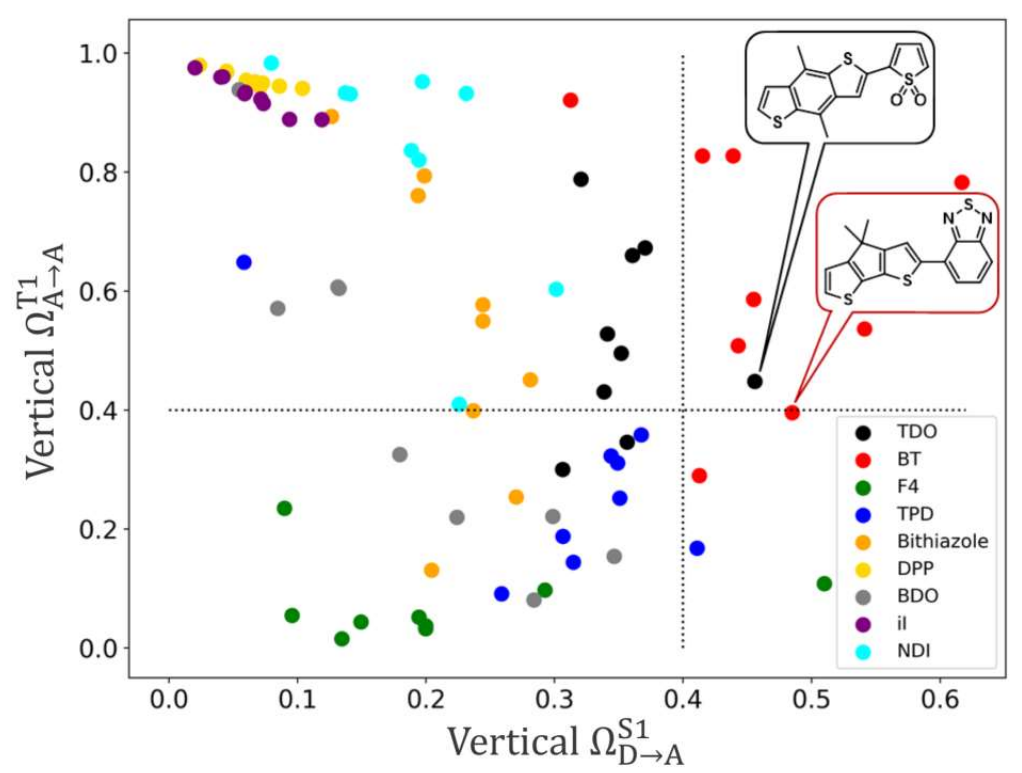

Figure 6: Donor-to-acceptor charge-transfer contribution of $\mathrm{S}_{1}\left(\Omega_{\mathrm{D} \rightarrow \mathrm{A}}^{\mathrm{S}},\right)$ and local acceptor contribution of $\mathrm{T}_{1}$ $\left(\Omega_{\mathrm{A} \rightarrow \mathrm{A}}^{\mathrm{T} 1}\right.$, ) obtained for the 81 dimers, coloured based on the acceptor. Evaluated with TheoDORE using results from computations with TDDFT (TDA) at $\omega \mathrm{B} 97 \mathrm{X}-\mathrm{D} / 6-31 \mathrm{G}^{*}$ level. 
Figure 8 associates the computed FMO ratios with the computed $\Omega_{\mathrm{D} \rightarrow \mathrm{A}}^{\mathrm{S} 1}$ values. It can be seen that the donor-acceptor monomer pairs with FMOs best suited for CT are located above 1.0 in both axes. In contrast, dimers with FMO ratios below 1 correctly predict minor CT character $\left(\Omega_{\mathrm{D} \rightarrow \mathrm{A}}^{\mathrm{S} 1}<0.2\right)$. This numerical comparison using monomer FMO energies is therefore a robust metric for eliminating poor potential iSF candidates.

To illustrate the direct impact of monomer FMO energies on $\Omega_{\mathrm{D} \rightarrow \mathrm{A}}^{\mathrm{S} 1}$, we generated 25 substituted bithiophene-BT donor-acceptor pairs. The monomer energy levels are substantially modulated through functionalization of the conjugated backbone with electron-donating (-OH), electron-withdrawing ($\mathrm{CN})$ and halide $(-\mathrm{F},-\mathrm{Cl})$ moieties. As a result, the CT character of $S_{1}$ in the dimer $\left(\Omega_{\mathrm{D} \rightarrow \mathrm{A}}^{\mathrm{S} 1}=0.44\right.$ when unsubstituted) becomes as low as 0.26 when an electron-withdrawing group is placed on the donor unit, and as high as 0.75 when electron-donating (withdrawing) groups are attached to the donor (acceptor) moiety. These variations are correctly captured by the FMO ratio model (Figure 8), which reproduces the general increase of $\Omega_{\mathrm{D} \rightarrow \mathrm{A}}^{\mathrm{S} 1}$ as the FMO ratio increases. This example demonstrates that monomer functionalization can be used to optimize the properties necessary for iSF.

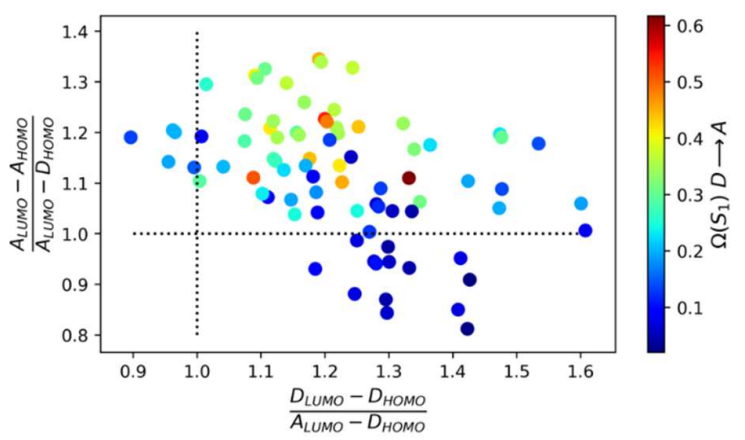

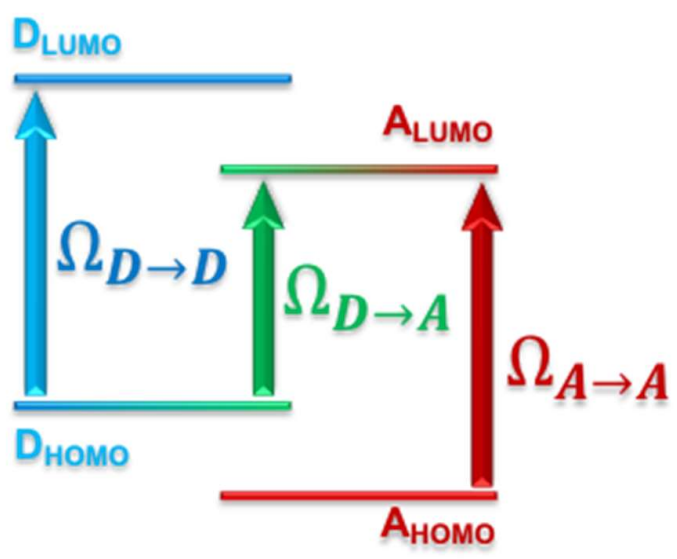

Donor Acceptor

Figure 7: Schematic representation of the dependence of $\Omega_{\mathrm{D} \rightarrow \mathrm{A}}^{\mathrm{S1}}$ in the dimer with the FMOs (HOMO, LUMO) of the monomers. The local excitations in the donor (blue) and acceptor (red) compete with the CT excitation (green).

Figure 8: (left) FMO ratios of the 81 donor-acceptor monomer pairs included in our dataset (see section 2.2), and (right) of 25 substituted bithiophene-BT monomer pairs. Cut-offs for discarding poor CT dimers are represented by the dotted lines. The structures of the reference (non-substituted) dimer and the dimers with the highest and lowest $\Omega_{\mathrm{D} \rightarrow \mathrm{A}}^{\mathrm{S} 1}$ values are shown. In both plots, the $\Omega_{\mathrm{D} \rightarrow \mathrm{A}}^{\mathrm{S1}}$ of the resulting dimer is given by the colour gradient. 


\section{SCREENING PROTOCOL}

The protocol established to evaluate and screen promising iSF candidates among donor-acceptor copolymers consists of the following steps:

Step 1. Compute the ground state FMOs of all donor and acceptor monomer cores, and evaluate the FMO ratios for the donor-acceptor dimers according to the expressions

$$
\frac{D_{\text {LUMO }}-D_{\text {HOMO }}}{A_{\text {LUMO }}-D_{\text {HOMO }}}>1 \text { and } \frac{A_{\text {LUMO }}-A_{\text {HOMO }}}{A_{\text {LUMO }}-D_{\text {HOMO }}}>1
$$

Step 2. For the candidate donor-acceptor combinations resulting from step 1 , generate the dimers and compute the vertical $S_{1}$ and $T_{1}$ excited energies. Apply the energetic criterion associated with vertical energies:

$$
\Delta E_{S T}^{\text {vert }} \geq-1 \mathrm{eV}
$$

Step 3. Determine the character of the vertical $S_{1}$ and $\mathrm{T}_{1}$ states, and apply the threshold for criteria 2 and 3 based on the following omega values:

$$
\Omega_{\mathrm{A} \rightarrow \mathrm{A}}^{\mathrm{T} 1} \geq 0.4 \text { and } \Omega_{\mathrm{D} \rightarrow \mathrm{A}}^{\mathrm{S} 1} \geq 0.4
$$

Until this point, the computations are limited to ground state (Step 1) and vertical excitations (Steps 2 and 3), and neither adiabatic computations nor structures larger than dimers are required. It is worth emphasizing that Step 1 significantly reduces the number of computations from $\mathrm{N}_{\mathrm{D}^{*}} \mathrm{~N}_{\mathrm{A}}$ to $\mathrm{N}_{\mathrm{D}}+\mathrm{N}_{\mathrm{A}}$ (where $\mathrm{N}_{\mathrm{D}}$ is number of donors and $\mathrm{N}_{\mathrm{A}}$ is number of acceptors). To illustrate the efficiency of this protocol in filtering candidates, we apply it to the dataset of 81 dimers generated in this work (section 2.2). First, 19 possible combinations (representing $23 \%$ of the dataset) would be eliminated in Step 1 and would not require dimer excited state computations. Then, 42 dimers $(52 \%)$ would be rejected in Step 2, and $16(20 \%)$ in Step 3. This leaves only four materials ( $5 \%$ of the initial dataset, Figure 9) for deeper study. Remarkably, two of these four candidates have already been reported to undergo iSF (BDT-TDO and CPDT-BT), supporting the convenience of this protocol. ${ }^{21,24}$ Note that the cut-off values used here were established at the $\omega \mathrm{B} 97 \mathrm{X}-\mathrm{D}$ level of theory, and that these parameters may shift depending on the functional used.
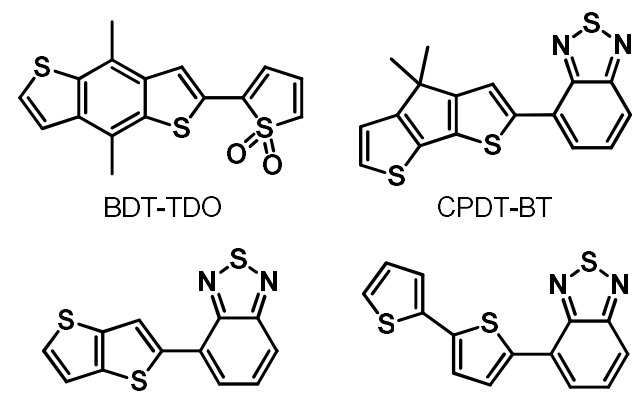

Thieno[3,2-b]thiophene-BT

2,2'-Bithiophene-BT

Figure 9: Four potential candidates for iSF, as identified by the present screening protocol.

\section{CONCLUSION}

We have developed a cost-effective computational protocol to perform large-scale screening of donoracceptor copolymers with promising features for intramolecular singlet-fission. Using a structurally diverse database of donor and acceptor units, we have established a simplified yet robust computational strategy to evaluate the energy splitting criterion and the charge-transfer requirements of the D-A candidates from conventional vertical excited state computations. In the context of accelerated screening, we have proposed an expression to predict the excited state character of D-A dimers from the FMO energies of their constituent donor and acceptor units. This drastically reduces computational time in initial screening stages, as the number of computations is reduced from $\mathrm{N}_{D^{*}} \mathrm{~N}_{\mathrm{A}}$ to $\mathrm{N}_{\mathrm{D}}+\mathrm{N}_{\mathrm{A}}, \mathrm{N}_{\mathrm{D}}$ and $\mathrm{N}_{\mathrm{A}}$ being the number of donor and acceptors considered.

This protocol correctly identified two donoracceptor pairs that exhibit singlet fission behaviour experimentally, ${ }^{21,24}$ and proposed two promising new candidates, thieno[3,2-b]thiophene-BT and 2,2'bithiophene-BT, that have not been studied to date. Benzothiadiazole (BT) in particular shows promise as an acceptor unit in iSF D-A materials. Altogether, these findings pave the way for high-throughput screening of large, chemically diverse databases of D-A conjugated polymers as a mean to bolster the collective library of SF materials. 


\section{ASSOCIATED CONTENT}

Details of database construction, benchmarking results, and supplementary figures pertaining to excited state character, tetramers, monomer bandgaps and excitonic effects are made available in the Supporting Information.

\section{DATASET}

Raw data used to generate all figures (dimer excitation energies and state character, monomer FMO energies and excitation energies, substituted monomer FMO energies, and substituted dimer excitation energies and state character) will be made available in a Materials Cloud repository upon publication. The collection of all output files from Gaussian, Turbomole and TheoDORE computations is available at the same location.

\section{AUTHOR INFORMATION}

\section{Corresponding author}

E-mail: clemence.corminboeuf@epfl.ch

\section{Orcid}

Maria Fumanal: 0000-0002-4175-8876

Sergi Vela: 0000-0002-3431-2470

Clémence Corminboeuf: 0000-0001-7993-2879

\section{Notes}

The authors declare no competing financial interest.

\section{ACKNOWLEDGMENTS}

The authors are grateful to the EPFL for financial support and the allocation of computational resources. M.F. and S.V. acknowledge funding from European Union's H2020 research and innovation, under the MSCA-IF-2018 (G.A. \#836849) and MSCA-IF-2017 programs (G.A. \#794519), respectively. The authors thank Raimon Fabregat for the TOC graphic.

\section{REFERENCES}

(1) Singh, S.; Jones, W. J.; Siebrand, W.; Stoicheff, B. P.; Schneider, W. G. Laser Generation of Excitons and Fluorescence in
Anthracene Crystals. J. Chem. Phys. 1965, 42, 330342.

(2) Casanova, D. Theoretical modeling of singlet fission. Chem. Rev. 2018, 118, 7164-7207.

(3) Smith, M. B.; Michl, J. Singlet fission. Chem. Rev. 2010, 110, 6891-6936.

(4) Fuemmeler, E. G.; Sanders, S. N.; Pun, A. B.; Kumarasamy, E.; Zeng, T.; Miyata, K.; Steigerwald, M. L.; Zhu, X.-Y.; Sfeir, M. Y.; Campos, L. M. A direct mechanism of ultrafast intramolecular singlet fission in pentacene dimers. ACS Cent. Sci. 2016, 2, 316-324.

(5) Beljonne, D.; Yamagata, H.; Brédas, J.; Spano, F.; Olivier, Y. Charge-transfer excitations steer the Davydov splitting and mediate singlet exciton fission in pentacene. Phys. Rev. Lett. 2013, 110, 226402.

(6) Berkelbach, T. C.; Hybertsen, M. S.; Reichman, D. R. Microscopic theory of singlet exciton fission. I. General formulation. J. Chem. Phys. 2013, 138, 114102.

(7) Berkelbach, T. C.; Hybertsen, M S.; Reichman, D. R. Microscopic theory of singlet exciton fission. II. Application to pentacene dimers and the role of superexchange. J. Chem. Phys. 2013, 138, 114103 .

(8) Chan, W.-L.; Berkelbach, T. C.; Provorse, M. R.; Monahan, N. R.; Tritsch, J. R.; Hybertsen, M. S.; Reichman, D. R.; Gao, J.; Zhu, X.-Y. The quantum coherent mechanism for singlet fission: Experiment and theory. Acc. Chem. Res. 2013, 46, 1321-1329.

(9) Singh, S.; Stoicheff, B. DoublePhoton Excitation of Fluorescence in Anthracene Single Crystals. J. Chem. Phys. 1963, 38, 20322033.

(10) Swenberg, C.; Ratner, M. A.; Geacintov, N. Energy dependence of optically induced exciton fission. J. Chem. Phys. 1974, 60, 2152-2157.

(11) Jundt, C.; Klein, G.; Sipp, B.; Le Moigne, J.; Joucla, M.; Villaeys, A. Exciton dynamics in pentacene thin films studied by pumpprobe spectroscopy. Chem. Phys. Lett. 1995, 241, 84-88.

(12) Chan, W.-L.; Ligges, M.; Jailaubekov, A.; Kaake, L.; Miaja-Avila, L.; Zhu, X.-Y. Observing the multiexciton state in singlet fission and ensuing ultrafast multielectron transfer. Science 2011, 334, 1541-1545.

(13) Dillon, R. J.; Piland, G. B.; Bardeen, C. J. Different rates of singlet fission in monoclinic versus orthorhombic crystal forms of diphenylhexatriene. J. Am. Chem. Soc. 2013, 135, 17278-17281.

(14) Johnson, J. C.; Nozik, A. J.; Michl, J. The role of chromophore coupling in singlet fission. Acc. Chem. Res. 2013, 46, 12901299. 
(15) Schwerin, A. F.; Johnson, J. C.; Smith, M. B.; Sreearunothai, P.; Popovic, D.; Černý, J. i.; Havlas, Z.; Paci, I.; Akdag, A.; MacLeod, M. K.; Chen, X.; David, D. E.; Ratner, M. A.; Miller, J. R.; Nozik, A. J.; Michl, J. Toward designed singlet fission: electronic states and photophysics of 1, 3-diphenylisobenzofuran. $J$. Phys. Chem. A 2010, 114, 1457-1473.

(16) Müller, A. M.; Avlasevich, Y. S.; Müllen, K.; Bardeen, C. J. Evidence for exciton fission and fusion in a covalently linked tetracene dimer. Chem. Phys. Lett. 2006, 421, 518-522.

(17) Müller, A. M.; Avlasevich, Y. S.; Schoeller, W. W.; Müllen, K.; Bardeen, C. J. Exciton fission and fusion in bis (tetracene) molecules with different covalent linker structures. J. Am. Chem. Soc. 2007, 129, 14240-14250.

(18) Sanders, S. N.; Kumarasamy, E.; Pun, A. B.; Steigerwald, M. L.; Sfeir, M. Y.; Campos, L. M. Intramolecular singlet fission in oligoacene heterodimers. Angew. Chem. Int. Ed. 2016, 55, 3373-3377.

(19) Zirzlmeier, J.; Lehnherr, D.; Coto, P. B.; Chernick, E. T.; Casillas, R.; Basel, B. S.; Thoss, M.; Tykwinski, R. R.; Guldi, D. M. Singlet fission in pentacene dimers. Proc. Natl. Acad. Sci. U.S.A 2015, 112, 5325-5330.

(20) Rademaker, H.; Hoff, A. J.; Van Grondelle, R.; Duysens, L. N. Carotenoid triplet yields in normal and deuterated Rhodospirillum rubrum. Biochim. Biophys. Acta, Bioenerg. 1980, 592, 240-257.

(21) Grancini, G.; Maiuri, M.; Fazzi, D.; Petrozza, A.; Egelhaaf, H.; Brida, D.; Cerullo, G.; Lanzani, G. Hot exciton dissociation in polymer solar cells. Nat. Mater. 2013, 12, 29.

(22) Kasai, Y.; Tamai, Y.; Ohkita, H.; Benten, H.; Ito, S. Ultrafast singlet fission in a push-pull low-bandgap polymer film. J. Am. Chem. Soc. 2015, 137, 15980-15983.

(23) Zhai, Y.; Sheng, C.; Vardeny, Z. $V$. Singlet fission of hot excitons in $\pi$-conjugated polymers. Philos. Trans. R. Soc. London, Ser. A 2015, 373, 20140327.

(24) Busby, E.; Xia, J.; Wu, Q.; Low, J. Z.; Song, R.; Miller, J. R.; Zhu, X. Y.; Campos, Luis M.; Sfeir, M. Y. A design strategy for intramolecular singlet fission mediated by chargetransfer states in donor-acceptor organic materials. Nat. Mater. 2015, 14, 426.

(25) Hu, J.; Xu, K.; Shen, L.; Wu, Q.; He, G.; Wang, J.-Y.; Pei, J.; Xia, J.; Sfeir, M. Y. New insights into the design of conjugated polymers for intramolecular singlet fission. Nat. Commun. 2018, 9, 2999.

(26) Padula, D.; Omar, Ö. H.;

Nematiaram, T.; Troisi, A. Singlet fission molecules among known compounds: finding a few needles in a haystack. Energ. Environ. Sci. 2019, $12,2412-2416$.
(27) Aryanpour, K.; Dutta, T.; Huynh, U. N.; Vardeny, Z. V.; Mazumdar, S. Theory of primary photoexcitations in donor-acceptor copolymers. Phys. Rev. Lett. 2015, 115, 267401.

(28) Paci, I.; Johnson, J. C.; Chen, X.; Rana, G.; Popović, D.; David, D. E.; Nozik, A. J.; Ratner, M. A.; Michl, J. Singlet fission for dyesensitized solar cells: Can a suitable sensitizer be found? J. Am. Chem. Soc. 2006, 128, 16546-16553.

(29) Zhou, H.; Yang, L.; You, W. Rational design of high performance conjugated polymers for organic solar cells. Macromolecules 2012, 45, 607-632.

(30) Guo, X.; Baumgarten, M.; Müllen, K. Designing $\pi$-conjugated polymers for organic electronics. Prog. Polym. Sci. 2013, 38, 1832-1908.

(31) Cheng, Y.-J.; Yang, S.-H.; Hsu, C.-S. Synthesis of conjugated polymers for organic solar cell applications. Chem. Rev. 2009, 109, 5868-5923.

(32) Pouliot, J.-R.; Grenier, F.; Blaskovits, J. T.; Beaupré, S.; Leclerc, M. Direct (Hetero)arylation Polymerization: Simplicity for Conjugated Polymer Synthesis. Chem. Rev. 2016, $116,14225-14274$.

(33) Blaskovits, J. T.; Leclerc, M. C $\square$ $\mathrm{H}$ Activation as a Shortcut to Conjugated Polymer Synthesis. Macromol. Rapid Commun. 2019, 40, 1800512

(34) Po, R.; Bernardi, A.; Calabrese, A.; Carbonera, C.; Corso, G.; Pellegrino, A. From lab to fab: how must the polymer solar cell materials design change? - an industrial perspective. Energ. Environ. Sci. 2014, 7, 925-943.

(35) Weininger, D. SMILES, a chemical language and information system. 1. Introduction to methodology and encoding rules. $J$. Chem. Inf. Comput. Sci. 1988, 28, 31-36.

(36) O'Boyle, N. M.; Banck, M.; James, C. A.; Morley, C.; Vandermeersch, T.; Hutchison, G. R. Open Babel: An open chemical toolbox. J. Cheminf. 2011, 3, 33.

(37) M. J. Frisch; G. W. Trucks; H. B. Schlegel; G. E. Scuseria; M. A. Robb; J. R. Cheeseman; G. Scalmani; V. Barone; G. A. Petersson; H. Nakatsuji; X. Li; M. Caricato; A. Marenich; J. Bloino; B. G. Janesko; R. Gomperts; B. Mennucci; H. P. Hratchian; J. V. Ortiz; A. F. Izmaylov; J. L. Sonnenberg; D. Williams-Young; F. Ding; F. Lipparini; F. Egidi; J. Goings; B. Peng; A. Petrone; T. Henderson; D. Ranasinghe; V. G. Zakrzewski; J. Gao; N. Rega; G. Zheng; W. Liang; M. Hada; M. Ehara; K. Toyota; R. Fukuda; J. Hasegawa; M. Ishida; T. Nakajima; Y. Honda; O. Kitao; H. Nakai; T. Vreven; K. Throssell; J. A. Montgomery, J.; J. E. Peralta; F. Ogliaro; M. Bearpark; J. J. Heyd; E. Brothers; K. N. Kudin; V. N. Staroverov; T. Keith; R. Kobayashi; J. Normand; K. Raghavachari; A. Rendell; J. C. 
Burant; S. S. Iyengar; J. Tomasi; M. Cossi; J. M. Millam; M. Klene; C. Adamo; R. Cammi; J. W.

Ochterski; R. L. Martin; K. Morokuma; O. Farkas; J. B. Foresman; Fox, D. J. Gaussian 16, Revision A.03 Gaussian, Inc., Wallingford CT. 2016.

(38) Peach, M. J.; Tozer, D. J. Overcoming low orbital overlap and triplet instability problems in TDDFT. J. Phys. Chem. A 2012, 116, 9783-9789.

(39) Laurent, A. D.; Jacquemin, D. TD-DFT benchmarks: a review. Int. J. Quantum Chem 2013, 113, 2019-2039.

(40) Adamo, C.; Jacquemin, D. The calculations of excited-state properties with TimeDependent Density Functional Theory. Chem. Soc. Rev. 2013, 42, 845-856.

(41) O'boyle, N. M.; Tenderholt, A. L.; Langner, K. M. Cclib: a library for packageindependent computational chemistry algorithms. J. Comput. Chem. 2008, 29, 839-845.

(42) Plasser, F.; Lischka, H. Analysis of excitonic and charge transfer interactions from quantum chemical calculations. J. Chem. Theory Comput. 2012, 8, 2777-2789.

(43) Plasser, F. TheoDORE 1.7: A package for theoretical density, orbital relaxation, and exciton analysis. TheoDORE 1.7: A package for theoretical density, orbital relaxation, and exciton analysis 2017.

(44) Chidthong, R.; Hannongbua, S.; Aquino, A. J.; Wolschann, P.; Lischka, H. Excited state properties, fluorescence energies, and lifetime of a poly (fluorene-pyridine) copolymer, based on TD-DFT investigation. J. Comput. Chem. 2007, 28, 1735-1742.

(45) Chen, T.; Zheng, L.; Yuan, J.;

An, Z.; Chen, R.; Tao, Y.; Li, H.; Xie, X.; Huang, $\mathrm{W}$. Understanding the control of singlet-triplet splitting for organic exciton manipulating: a combined theoretical and experimental approach. Sci. Rep. 2015, 5, 10923. 